\title{
Improvement of the Digestibility of Sulfated Hyaluronans by Bovine Testicular Hyaluronidase: A UV Spectroscopic and Mass Spectrometric Study
}

\author{
Katharina Lemmnitzer, ${ }^{1}$ Jürgen Schiller, ${ }^{1}$ Jana Becher, ${ }^{2}$ \\ Stephanie Möller, ${ }^{2}$ and Matthias Schnabelrauch ${ }^{2}$ \\ ${ }^{1}$ Institute of Medical Physics and Biophysics, Medical Faculty, University of Leipzig, Härtelstraße 16-18, 04107 Leipzig, Germany \\ ${ }^{2}$ INNOVENT e. V., Biomaterials Department, Pruessingstraße 27B, 07745 Jena, Germany
}

Correspondence should be addressed to Jürgen Schiller; juergen.schiller@medizin.uni-leipzig.de

Received 27 January 2014; Accepted 24 April 2014; Published 27 May 2014

Academic Editor: Daniel Huster

Copyright ( 2014 Katharina Lemmnitzer et al. This is an open access article distributed under the Creative Commons Attribution License, which permits unrestricted use, distribution, and reproduction in any medium, provided the original work is properly cited.

\begin{abstract}
Glycosaminoglycans (GAGs) such as hyaluronan (HA) and chondroitin sulfate (CS) are important, natural polysaccharides which occur in biological (connective) tissues and have various biotechnological and medical applications. Additionally, there is increasing evidence that chemically (over)sulfated GAGs possess promising properties and are useful as implant coatings. Unfortunately, a detailed characterization of these GAGs is challenging: although mass spectrometry (MS) is one of the most powerful tools to elucidate the structures of (poly)saccharides, MS is not applicable to high mass polysaccharides, but characteristic oligosaccharides are needed. These oligosaccharides are normally generated by enzymatic digestion. However, chemically modified (particularly sulfated) GAGs are extremely refractive to enzymatic digestion. This study focuses on the investigation of the digestibility of GAGs with different degrees of sulfation by bovine testicular hyaluronidase (BTH). It will be shown by using an adapted spectrophotometric assay that all investigated GAGs can be basically digested if the reaction conditions are carefully adjusted. However, the oligosaccharide yield correlates reciprocally with the number of sulfate residues per polymer repeating unit. Finally, matrix-laser desorption and ionization (MALDI) MS will be used to study the released oligosaccharides and their sulfation patterns.
\end{abstract}

\section{Introduction}

Polysaccharides of the glycosaminoglycan (GAG) type such as hyaluronan (HA) or chondroitin sulfate (CS) are important constituents of the extracellular matrix (ECM) of connective tissues which is omnipresent in all vertebrates [1]. Since many inflammatory diseases such as rheumatoid arthritis are accompanied by degradation of the ECM, GAGs experience nowadays considerable medical and diagnostic interest [2]. Advances in "tissue engineering," a hot topic of current biomedical research, have also significantly increased the scientific interest in GAGs [3].

Motivated by the discovery of the "sulfation code" [4], the interest in chemically (over)sulfated GAGs has also significantly increased because these compounds were shown to possess many beneficial properties and improve, for instance, the incorporation of metal implants into the corresponding tissue such as bone [5]. However, there are also negative aspects: chemically sulfated GAGs may be toxic, as evidenced by the lethal contamination of heparin with a chemically oversulfated CS [6], so caution is advised prior to the in vivo use of any chemically sulfated GAGs, including sulfated HA.

Although the overall degree of sulfation can be easily determined by elementary analysis (i.e., by the determination of the sulfur content of a GAG sample), a more detailed structural analysis of the sulfation pattern is a challenging task. Although ${ }^{13} \mathrm{C}$ nuclear magnetic resonance (NMR) spectroscopy is one of the most powerful methods of structural GAG analysis, this method may easily fail for two reasons. First, the line widths of the ${ }^{13} \mathrm{C}$ NMR resonances of GAG polysaccharides are significant and, thus, prevent the detection of small chemical shift differences [1]. Second, the 
introduction of sulfate residues reduces the achievable frequency dispersion of the carbon resonances of the dedicated GAG and, thus, causes considerable assignment problems [7].

Even though mass spectrometry (MS) is an additional powerful method of structure elucidation (particularly when combined with MS/MS and/or chromatographic separation) [5], the application of MS to polysaccharides is difficult and this particularly applies if polymers with charged functional groups such as sulfate (i.e., strong electrolytes) are of interest: while native dextran (with a molecular weight of about $67 \mathrm{kDa}$ but without any charged groups) [8] is detectable by MS, the strongly sulfated heparin was not detectable in a mass higher than about $4 \mathrm{kDa}$ [5].

Therefore, the most common way of mass spectrometric GAG analysis is based on previous enzymatic digestion of the polysaccharides: using enzymes such as testicular or bacterial hyaluronidases or bacterial chondroitinases (normally of the ABC type which is capable of cleaving CS A, B, and C), the native GAGs can be efficiently converted into characteristic oligosaccharides [1]. While chondroitinase ABC generates nearly exclusively a single $\Delta^{4,5}$ unsaturated disaccharide [9], the mechanisms of the hyaluronidase digest are more complex leading to a broad mixture of different oligosaccharides [10], whereby the hexasaccharide is normally the most abundant product [11]. The efficient digestibility of the naturally occurring GAGs is the most important reason why the corresponding oligosaccharides (particularly that of HA) are commercially available at moderate prices. Higher sulfated GAG oligosaccharides (with the exception of heparin) are, however, commercially less readily available. This is very unfortunate because such defined GAG oligosaccharides would be needed for several reasons: on the one hand, such compounds are required for binding studies with other molecules, particularly proteins, to reduce the complexity (in comparison to the native GAGs) of the system and to enable molecular modeling [12]. On the other hand, it has been recently shown that GAGs exhibit different properties depending on their molecular weights: while high mass HA has anti-inflammatory properties, HA oligosaccharides are known to exhibit proinflammatory properties, maybe due to the enhanced content of reactive end groups [13].

There are two reasons why sulfated oligosaccharides are commercially scarcely available. First, (over)sulfated GAGs (that are available from some animal tissues and/or can be synthesized by chemical sulfation of the native GAG polysaccharides) are less efficiently digested by enzymes such as chondroitinase or hyaluronidase [14]. Second, the chromatographic separation of mixtures of variously (over)sulfated GAGs is very challenging and cannot (to these authors' best knowledge) be routinely performed in larger quantities, even if the separation of native HA or CS oligosaccharides by liquid chromatography or capillary electrophoresis is not a major problem at all [15].

We will focus here particularly on the first problem: by using an adapted spectrophotometric assay as well as matrix-assisted laser desorption and ionization time-of-flight (MALDI-TOF) MS, we will show that enzymatic digestion of all (over)sulfated GAGs can be basically achieved if the reaction conditions are carefully adjusted. Nevertheless, the yield of digestion products decreases if the degree of sulfation of the polysaccharide educt increases.

\section{Materials and Methods}

2.1. Chemicals. Hyaluronan (HA, from Streptococcus, $\mathrm{MW} \approx$ $1.1 \times 10^{6} \mathrm{~g} \mathrm{~mol}^{-1}$ ) was obtained from Aqua Biochem Dessau, Germany; sulfur trioxide/dimethylformamide complex $\left(\mathrm{SO}_{3}\right.$-DMF, purum, $\geq 97 \%$, active $\mathrm{SO}_{3} \geq 48 \%$ ) and sulfur trioxide/pyridine complex ( $\mathrm{SO}_{3}$-pyridine, pract.; $\geq 45 \%$ $\mathrm{SO}_{3}$ ) were from Fluka Chemie, Buchs, Switzerland. All other chemicals, solvents, and hyaluronidases from bovine testes (BTH; Type I-S, lyophilized powder, 400-1000 units/mg solid) were obtained in highest commercially available purity from Sigma-Aldrich (Deisenhofen, Germany) and used without further purification.

\subsection{Synthesis and Analytical Characterization of Hyaluronan} Sulfates. The hyaluronan sulfates (sHA1-sHA3) were synthesized as previously described by Hintze et al. [16]. In brief, in a first step the sodium salt of HA was transformed into its tetrabutylammonium salt (TBA-HA) using a Dowex WX8 ion exchanger. The following sulfation reactions of the TBA-HA were performed under argon in DMF at room temperature. For the syntheses of sHAl (average number of sulfate groups per disaccharide repeating unit $\left(\mathrm{ds}_{\mathrm{s}}\right)=1.2$ ) and sHA2 $\left(\mathrm{ds}_{\mathrm{s}}=1.8\right)$ an $\mathrm{SO}_{3}$-pyridine complex (sHA1: molar polymer $/ \mathrm{SO}_{3}$ ratio 1:6; sHA2: molar polymer $/ \mathrm{SO}_{3}$ ratio 1:8) was used as sulfation agent. $\mathrm{sHA} 3\left(\mathrm{ds}_{\mathrm{s}}=3.0\right)$ was sulfated using an $\mathrm{SO}_{3}$-DMF complex (molar polymer/SO $\mathrm{SO}_{3}$ ratio $1: 20$ ). The sulfated products were isolated from the reaction mixture by precipitation into acetone and neutralized with ethanolic $\mathrm{NaOH}$ solution. The formed sodium salts of the sHAs were washed with acetone and purified by dialysis against distilled water followed by lyophilization and drying of the resulting polymers under vacuum.

The degree of sulfation was determined by estimation of the sulfur content using an automatic elemental analyzer (Euro EA3000 CHNS, EuroVector, Redavalle, Italy). Molecular weight determination was performed by gel permeation chromatography (GPC) with a double detection system consisting of a Postnova Analytics PN $3000\left(15^{\circ}\right)$ laser-light scattering (LLS) detector and a Jasco RID-1531 refraction index (RI) detector. Absolute values of number-average (Mn) and weight-average $(\mathrm{Mw})$ molecular weights were determined using the laser-light scattering (LLS) detection system. The calculation of the polydispersity $(\mathrm{PD}=\mathrm{Mw} / \mathrm{Mn})$ was performed on the basis of $\mathrm{Mn}$ and $\mathrm{Mw}$ values obtained from RI detection. GPC operating parameters can be found in the work by Hintze et al. [16]. The most relevant analytical data are also summarized in Table 1.

\subsection{Enzymatic Digestion of Differently Sulfated Hyaluro-} nan Derivatives. Aqueous solutions of (a) native, that is, nonsulfated hyaluronan, and (b) three chemically sulfated hyaluronan derivatives with degrees of sulfation of 1.2, 1.8, and 3.0 were digested with hyaluronidase from bovine testis 
TABLE 1: Selected characteristics of the synthesized HA derivatives.

\begin{tabular}{lccc}
\hline Sample & sHA1 & sHA2 & sHA3 \\
\hline $\mathrm{ds}_{\mathrm{s}}$ & 1.2 & 1.8 & 3.0 \\
$M_{n}\left(\mathrm{~g} \times \mathrm{mol}^{-1}\right)$ & $15910(39540)$ & $16673(43993)$ & $23725(29275)$ \\
$M_{w}\left(\mathrm{~g} \times \mathrm{mol}^{-1}\right)$ & $26790(87410)$ & $32370(86150)$ & $29525(48340)$ \\
$\mathrm{PD}$ & 2.2 & 2.0 & 1.7 \\
\hline
\end{tabular}

$\mathrm{ds}_{\mathrm{s}}$, number-average $\left(M_{n}\right)$, and weight-average $\left(M_{w}\right)$ molecular weights as determined by laser-light scattering (LLS) and refractive index (RI) (in parentheses) detection. Molecular weight distributions (polydispersity index $(\mathrm{PD})$ ) were determined based on the values calculated from RI detection.

(BTH) using a final concentration of $0.4 \mathrm{mg} / \mathrm{mL}$. The HA substrates $(0.5 \mathrm{mg} / \mathrm{mL}, 0.5 \mathrm{~mL})$ were dissolved in citratephosphate-buffer, consisting of $0.1 \mathrm{M}$ citric acid adjusted with $0.2 \mathrm{M}$ aqueous $\mathrm{Na}_{2} \mathrm{HPO}_{4}$ to the desired $\mathrm{pH}$ value of $\mathrm{pH}$ 5.7, and digested under continuous shaking for 20 hours at $37^{\circ} \mathrm{C}$. For further optimization of the digestion conditions the sodium chloride concentration was varied between 0 and $0.5 \mathrm{M}$ and the $\mathrm{pH}$ value between 3 and 9 .

\subsection{Determination of the Reactive End Groups by Photometry.} The number of reducing ( $\mathrm{N}$-acetylglucosamine) end groups was used as a measure of the extent of the enzymatic digestion: when a GAG is digested, the absolute amount of the GAG remains indeed constant. However, the number of molecules increases. Each of the resulting oligosaccharides possesses a reducing end group and the number of the end groups can be conveniently determined. For the performing of the Reissig method $[17,18]$ two solutions were prepared. Solution (I) contained $4.94 \mathrm{~g} \mathrm{H}_{3} \mathrm{BO}_{3}$ and $1.98 \mathrm{~g}$ $\mathrm{KOH}$ in $100 \mathrm{~mL}$ distilled water. Solution (II) comprised $5 \mathrm{~g} \mathrm{p}-$ dimethylaminobenzaldehyde (DMAB) dissolved in $6.25 \mathrm{~mL}$ $\mathrm{HCl}(12 \mathrm{M})$. This solution was made up to $50 \mathrm{~mL}$ with glacial acetic acid in a graduated cylinder and once again diluted 1:10 (v/v) with glacial acetic acid immediately prior to use. The relative amounts of the digestion products indicated by the Reissig signal (absorbance at $585 \mathrm{~nm}$ ) were monitored by a colorimetric assay according to Muckenschnabel et al. [18] modified by Asteriou et al. [19] and further modified in the following way: $100 \mu \mathrm{L}$ of the digestion mixture (or the corresponding polysaccharide solution in the absence of the enzyme) was mixed with $200 \mu \mathrm{L}$ of solution (I) to obtain a $\mathrm{pH}$ value of about 9 which is required for the MorganElson reaction (described in detail in [18]). After heating at $95^{\circ} \mathrm{C}$ for 3 minutes the samples were cooled to $4^{\circ} \mathrm{C}$. Afterwards, $1.5 \mathrm{~mL}$ solution (II) was added, thoroughly mixed, and placed in a shaker at $37^{\circ} \mathrm{C}$ for $15 \mathrm{~min}$. The absorbance at $585 \mathrm{~nm}$ was subsequently determined on a Hitachi U2000 spectrophotometer in $1.5 \mathrm{~mL}$ semimacro PMMA (polymethyl-methacrylate) cuvettes against water. The absorbance at $585 \mathrm{~nm}$ was also determined for the same sample but without previous digestion and subtracted from the value determined in the presence of the enzyme. This difference is referred to as the Reissig signal and is directly proportional to the number of reducing $\mathrm{N}$-acetyl-D-glucosamine end groups. The absorbance at $600 \mathrm{~nm}$ was determined as well to exclude potential influences of turbidity changes due to the digestion of long polysaccharide chains which may aggregate with the enzyme [20].

2.5. MALDI-TOF Mass Spectrometry. All MALDI-TOF mass spectra were acquired on an Autoflex mass spectrometer (Bruker Daltonics, Bremen, Germany) in the linear mode under delayed extraction conditions as previously described $[10,21]$. Although mass spectra recorded in the linear mode have only limited resolution and a reduced mass accuracy [21], the higher sensitivity and the reduced generation of fragmentation products are clear advantages of this approach. The system utilizes a pulsed nitrogen laser, emitting at $337 \mathrm{~nm}$. The extraction voltage was $20 \mathrm{kV}$ and gated matrix suppression was applied to prevent the saturation of the detector by matrix ions [22].

200 single laser shots were averaged for each mass spectrum. The laser fluence was kept about five percent above threshold to obtain optimum signal to noise ratios.

Saturated 9-aminoacridine (9-AA) $[23,24]$ in methanol was used as matrix for negative ion detection. Spectra were analyzed with the program FlexAnalysis.

\section{Results and Discussion}

The GAGs of particular interest for this communication are (a) native (nonsulfated) hyaluronan and (b) chemically sulfated HA derivatives. However, the polymer repeating units remain unchanged upon derivatization: the disaccharide unit of HA is composed of a D-glucuronic acid, which is 1,3-glycosidically linked to N-acetyl-D-glucosamine. These disaccharides are 1,4-glycosidically linked with each other forming a linear (nonbranched) polysaccharide with a molecular weight (depending on the biological source the HA sample is isolated from) reaching up to several hundred kilodalton $(\mathrm{kDa})$ [25]. The chemical sulfation is known to introduce additional sulfate residues preferentially at the C-6 of the N-acetyl-D-glucosamine unit and (under more vigorous reaction conditions) at the $\mathrm{C}-4$ of the latter unit and the C-2 and C-3 of the D-glucuronic acid residue. The number of sulfate residues per disaccharide unit depends on the reaction conditions and may be up to four. This is termed $\mathrm{ds}_{\mathrm{s}}=4$ and " $\mathrm{ds}_{\mathrm{s}}$ " denotes the average number of sulfate groups per disaccharide repeating unit. We will use here HA derivatives with a reduced extent of sulfation.

Figure 1 shows a schematic survey of the structures of the GAGs relevant to this study. The analytical data of the synthesized hyaluronan sulfates are summarized in Table 1.

Hyaluronidase from bovine testis (BTH) is known to act as a hydrolase and cleaves the 1,4-glycosidic linkages in the hyaluronan polysaccharide nearly randomly [26]. We will focus on this enzyme because BTH is commercially available at a very moderate price. Additionally, BTH also hydrolyses chondroitin, chondroitin-4-sulfate and chondroitin-6sulfate, as well as dermatan (sulfate) and its application is, thus, not limited to the degradation of HA [27].

The Reissig signal $[17,18]$ as a measure of the number of free N-acetyl-D-glucosamine end groups was determined before and subsequent to enzymatic digestion of the differently sulfated HA samples. In order to make the data more 


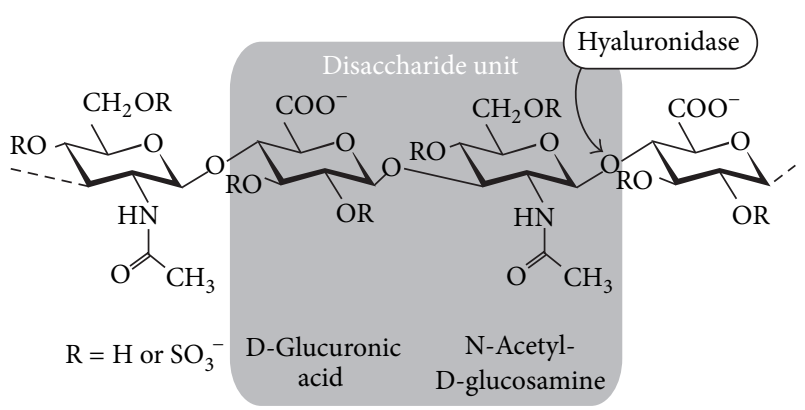

FIGURE 1: Chemical structure of native $(\mathrm{R}=\mathrm{H})$ and chemically sulfated $\mathrm{HA}\left(\mathrm{R}=\mathrm{SO}_{3}{ }^{-}\right)$. The cleavage site of hyaluronidase (exclusively the 1-4 glycosidic linkages) is also illustrated in the figure. Note that the sulfate residue represents a strong electrolyte; that is, it always exists in the deprotonated form.

obvious, the difference ( $\Delta$-absorbance) between both values at $585 \mathrm{~nm}$ will be shown as a relative measure of the extent of the related enzymatic activity

Figure 2 clearly indicates that the change in the absorbance at $585 \mathrm{~nm}$ depends significantly on the substrate and, thus, the degree of sulfation of the used polysaccharide: the digestion of the native HA results in the highest increase of the absorbance; that is, a maximum of oligosaccharides is obtained under these conditions. The sulfated HA derivatives with $\mathrm{ds}_{\mathrm{s}}=1.2$ (sHA1) and 1.8 (sHA2) are digested (within the experimental error range) to the same extents; however, only about $20 \%$ of the effect that is detectable in the case of the native HA can be achieved. That is, the extent of digestion is significantly reduced when sulfated HA samples are used as substrates $[5,14]$. Considering the accuracy of the applied method, the very low absorbance detected in the case of the highly sulfated derivative ( $\mathrm{sHA} 3, \mathrm{ds}_{\mathrm{s}}=3$ ) suggests that no significant degradation has taken place. Therefore, BTH is only capable of digesting the moderately sulfated HAs albeit only much smaller yields of oligosaccharides can be obtained when compared to the native HA.

The exhaustive enzymatic digestion of (sulfated) HAs leads to tetra-, hexa-, and octasaccharides as the most abundant products [10]. Of course, these products can be easily differentiated depending on the number of sulfate residues per oligosaccharide, that is, by their molecular weights. Table 2 shows the (theoretical) atomic masses of HA oligosaccharides generated from polymeric HA by digestion with BTH. In addition to nonsulfated HA, sulfated HA derivatives with varying numbers of sulfate groups are also considered in Table 2.

Soft ionization mass spectrometric techniques such as MALDI or ESI are convenient methods to investigate the molecular weights of oligosaccharides [28]. We will focus here on MALDI MS because this method is influenced to a lesser extent by sample impurities, particularly the salt content. In the negative ion MALDI-TOF mass spectra shown in Figure 3 all detected peaks can be easily assigned to the corresponding oligosaccharides (cf. Table 2). As expected, native HA is primarily digested into the tetra-, hexa-, and octasaccharides which can be easily differentiated (cf. trace

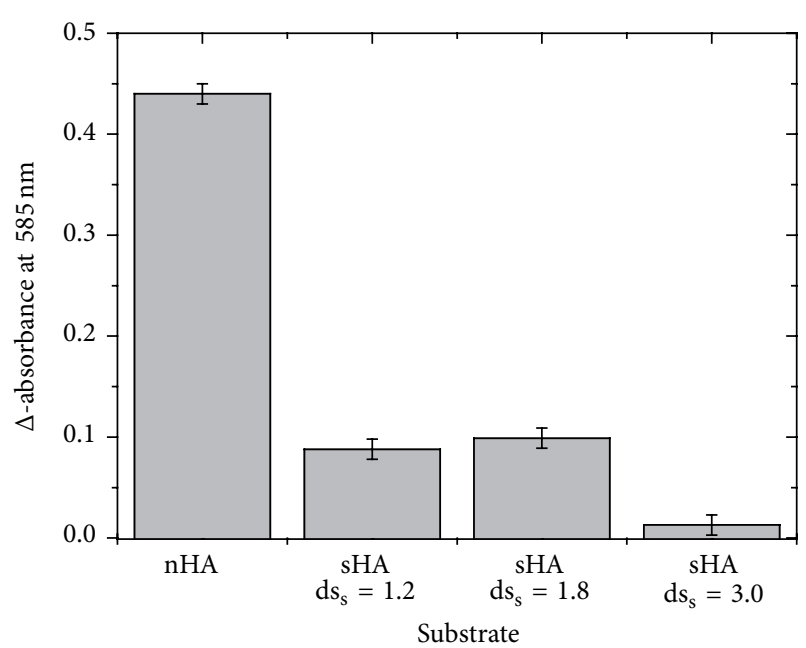

FIGURE 2: Dependence of the Reissig absorption (difference of the absorption at $585 \mathrm{~nm}$ without and subsequent to digestion with $\mathrm{BTH})$ on the used hyaluronan samples. All samples were digested with BTH for 20 hours at $37^{\circ} \mathrm{C}$ at $\mathrm{pH}$ 5.7. No salts beside the buffer components were added. For details see text.

TABLE 2: Mass list of the most abundant digestion products of HA after exhaustive digestion with bovine testicular hyaluronidase.

\begin{tabular}{lcccc}
\hline & \multicolumn{5}{c}{ Monoisotopic molecular masses } \\
Hyaluronan oligosaccharide & \multicolumn{4}{c}{ Number of sulfate residues } \\
& 0 & 1 & 2 & 3 \\
\hline Tetrasaccharide (HA-4) & 776.23 & 856.19 & 936.15 & 1016.10 \\
Hexasaccharide (HA-6) & 1155.34 & 1235.30 & 1315.26 & 1395.22 \\
Octasaccharide (HA-8) & 1534.46 & 1614.41 & 1694.37 & 1774.33 \\
\hline
\end{tabular}

The table includes the masses of sulfated derivatives with up to three sulfate residues per oligosaccharide. Please note that all data were calculated by using the monoisotopic masses. Since the data will be used to explain the mass spectra (vide infra), charge compensation by protonation (not by alkali metal ions) is exclusively assumed to occur.

(a) of Figure 3). The mass difference of one unit in comparison to the data given in Table 2 is caused by the loss of one proton which is necessary to make the oligosaccharide detectable as a negative ion.

In the case of the sulfated HA with $\mathrm{ds}_{\mathrm{s}}=1.2$ and 1.8 , however, only sulfated oligosaccharides are detectable, whereby the tetra-, hexa-, and octasaccharides with one to a maximum of three sulfate residues (cf. Figure 3, traces (b) and (c)) can be identified. The observed mass differences of 22 or 44 units (cf. Table 2) are caused by $\mathrm{H}^{+} / \mathrm{Na}^{+}$exchange. These differences do exclusively occur if the spectra of sulfated HA oligosaccharides are considered. Although a detailed investigation of this aspect is beyond the scope of this paper, this difference might be caused by either (a) the strongly different $\mathrm{pK}$ values of sulfate in comparison to carboxylate [1] or (b) an enhanced salt content within the chemically modified HA samples.

Unfortunately, the digestion of the HA sample with the most significant extent of sulfation $\left(\mathrm{sHA}_{3}, \mathrm{ds}_{\mathrm{s}}=3.0\right)$ did not result in an oligosaccharide concentration which could be 


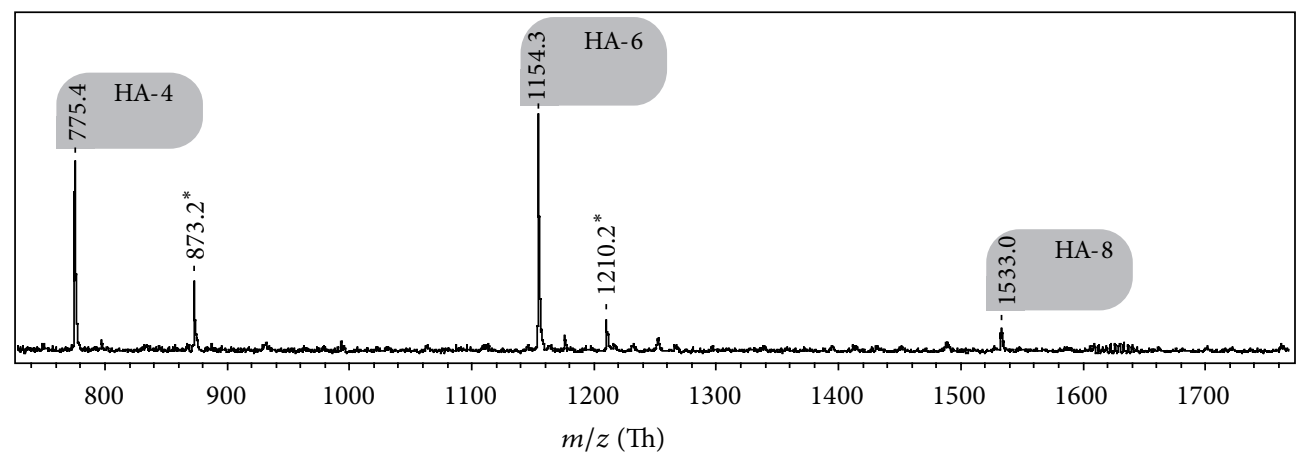

(a)

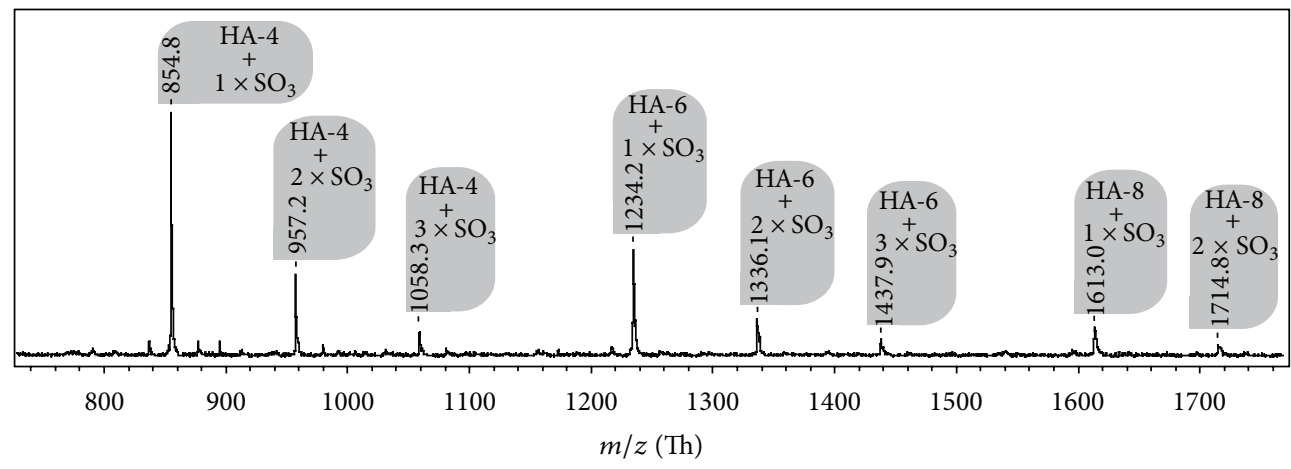

(b)

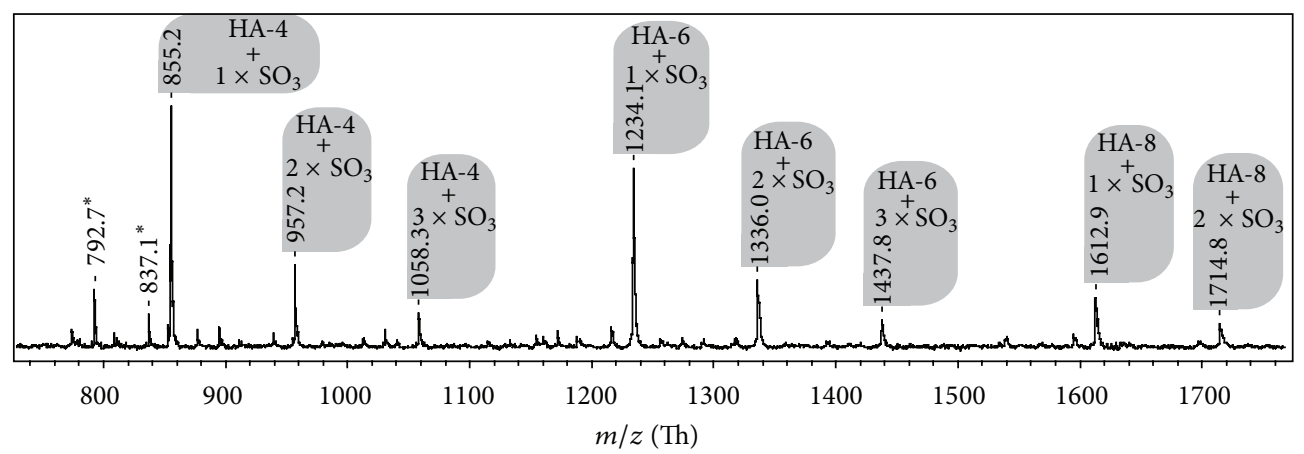

(c)

FIGURE 3: Negative ion MALDI-TOF mass spectra of differently sulfated HA samples subsequent to digestion with hyaluronidase but without any further purification of the reaction mixture. Trace (a) corresponds to native HA, while (b) and (c) represent HA with ds $\mathrm{s}_{\mathrm{s}}=1.2$ and 1.8 , respectively. All spectra were recorded in the linear mode of the MS device and in the presence of 9-aminoacridine as matrix with a sample to matrix ratio of 2 to $1(\mathrm{v} / \mathrm{v})$. This corresponds to a 25 -fold weight excess of the matrix. Peaks stemming from impurities are marked by asterisks. Please note that the achievable mass accuracy is only of the order of about $200 \mathrm{ppm}$. This is a rather typical value if the linear (but not the reflector modus) is used and the reason why only one decimal is given.

detected by MALDI MS. However, it should be explicitly noted that oligosaccharide concentrations below the detection limits are only one potential explanation: in addition to this aspect, charged saccharides are always more difficult to detect by means of MS than neutral saccharides. This particularly applies to carbohydrates with strong electrolytes such as sulfate [29].

In order to improve the extent of the enzymatic degradation of the HA with the most significant extent of sulfation, the digestion conditions were further optimized: the dependence of the Reissig signal (subsequent to BTH digestion) on the used $\mathrm{NaCl}$ concentration is shown in Figure 4. It is obvious that the maximum of digestion products is obtained when $0.15 \mathrm{M} \mathrm{NaCl}$ is used, that is, when the digestion is performed at physiological salt concentrations. This improved digestibility is presumably caused by the (partial) shielding of the negative charges of the HA polysaccharide by the sodium ions.

In addition to the salt concentration, the $\mathrm{pH}$ of the reaction mixture was also systematically varied to evaluate the optimum conditions of the enzymatic digestion, whereby the $\mathrm{NaCl}$ concentration was kept constant at $0.15 \mathrm{M}$. The achieved data (given in Figure 5) unequivocally indicate that a $\mathrm{pH}$ 


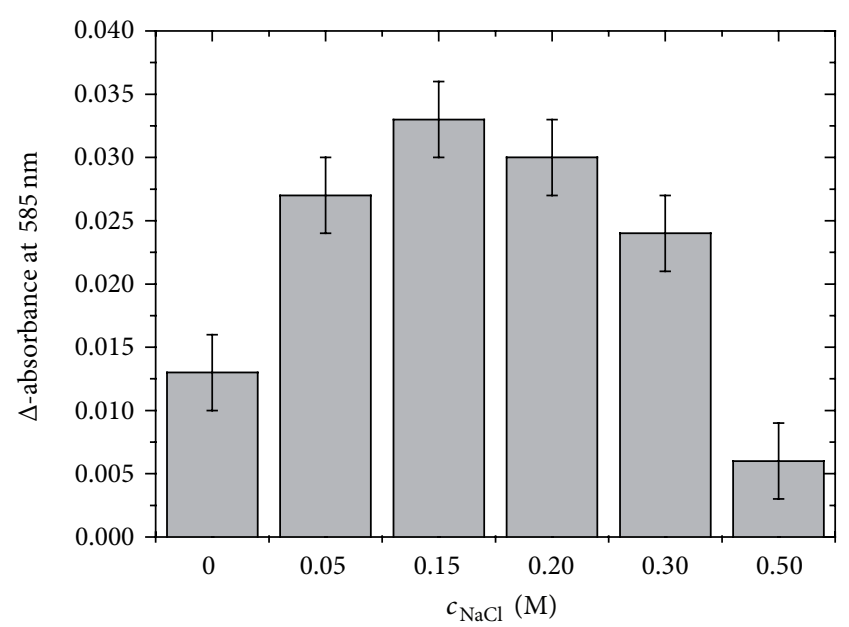

FIGURE 4: Dependence of the Reissig absorption (as a measure of the reducing end groups, i.e., the extent of the enzymatic digestion) on the $\mathrm{NaCl}$ concentration. Since the HA sample with the highest sulfate content $\left(\mathrm{ds}_{\mathrm{s}}=3\right)$ was most refractive to the hyaluronidase digestion, only this sample was investigated. All samples were digested with $\mathrm{BTH}$ for 20 hours at $37^{\circ} \mathrm{C}$ at $\mathrm{pH} 5.7$ prior to measurements.

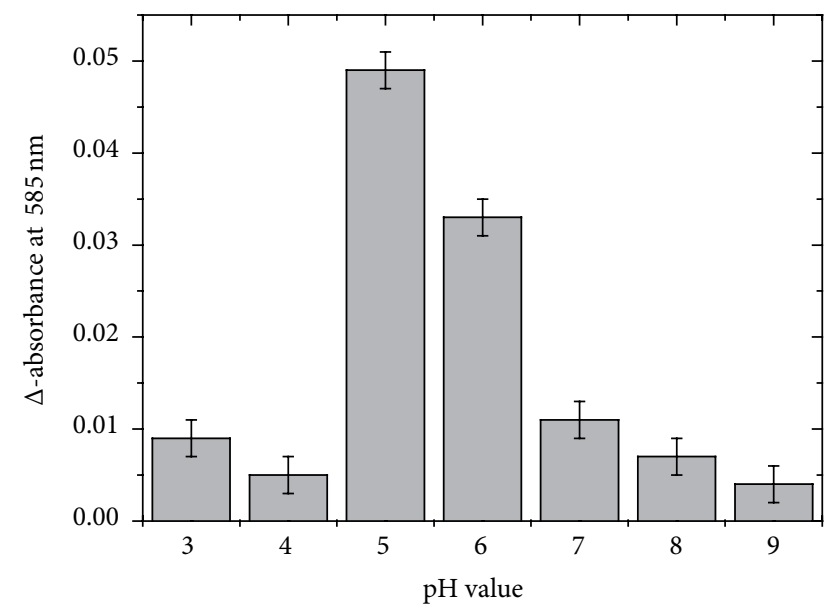

FIGURE 5: Dependence of the Reissig absorption on the $\mathrm{pH}$ value of the digestion mixture. Since the HA sample with the highest sulfate content (sHA3) was most refractive to the hyaluronidase digestion, only this sample was investigated. All samples were digested with $\mathrm{BTH}$ for 20 hours at $37^{\circ} \mathrm{C}$ and physiological $\mathrm{NaCl}$ concentration $(0.15 \mathrm{M})$.

value of about 5 represents the optimum reaction conditions to obtain a maximum of enzymatic digestion products, while reduced product yields are obtained at slightly lower or higher $\mathrm{pH}$ values.

All data related to the digestion of the HA sample with the highest sulfate content (sHA3) are finally summarized in Figure 6.

The comparison of the Reissig signal intensities after the three different digestions of the highly sulfated HA derivative (Figure 6) provides clear evidence that the yield of the oligosaccharides can be fourfold increased in comparison to

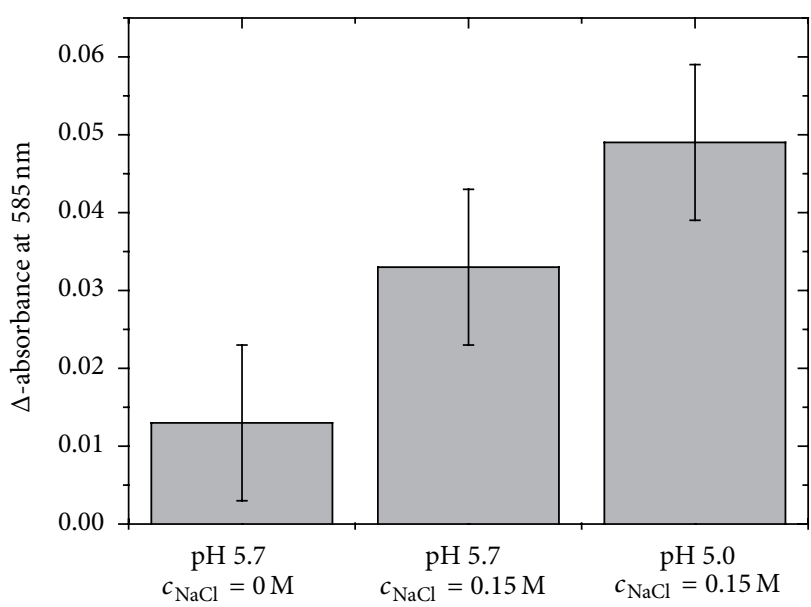

Figure 6: Determination of the Reissig absorption under the optimized reaction conditions discussed above. HA with ds $=3$ was exclusively used. The applied conditions are indicated directly in the figure. Error bars represent the systematic error of the performed measurements.

the originally used conditions (no $\mathrm{NaCl}$ addition and a $\mathrm{pH}$ of 5.7) if a sodium chloride concentration of $0.15 \mathrm{M}$ and a $\mathrm{pH}$ value of 5 are applied to digest the HA samples.

Although this might be considered as a minor progress only, this yield difference is sufficiently pronounced to enable the MALDI MS characterization of the related oligosaccharides: the negative ion MALDI-TOF mass spectrum of the HA polysaccharide with the most pronounced sulfate content $\left(\mathrm{ds}_{\mathrm{s}}=3.0\right)$ subsequent to BTH digestion at the optimized conditions is shown in Figure 7. In addition to the products already illustrated in the context of Figure 3, additional peaks at $m / z 1262.6$ and 1363.7 are obvious. Although these peaks possess minor intensities and there is slight interference with the corresponding hexasaccharide, these signals can be easily assigned to the HA tetrasaccharide with 5 and 6 sulfate residues, respectively.

The reader should note that the peak intensities do not necessarily correlate with the concentrations of the individual sulfated oligosaccharides [22]: in addition to the molecular weight of a compound, the number of the sulfate residues does also (negatively) affect the peak intensities. An additional problem is that there is a pronounced tendency of the sulfate residues to be lost in the gas phase [30] and the probability of sulfate loss increases with the number of sulfate residues. This problem can be minimized but never completely suppressed. Since electrospray ionization (ESI) MS is known to represent an even more gentle ionization technique than MALDI MS [31], we have also attempted to use ion trap (IT) ESI MS for the characterization of the individual samples. However, these attempts were so far not successful. We believe that the salt content (even subsequent to dilution) of the samples suppresses the successful ion generation within the ESI source because ESI MS is much more sensitive to contaminations when compared to MALDI MS [5].

Although we are aware of these obvious problems, the present study should be considered as a pioneering work: 


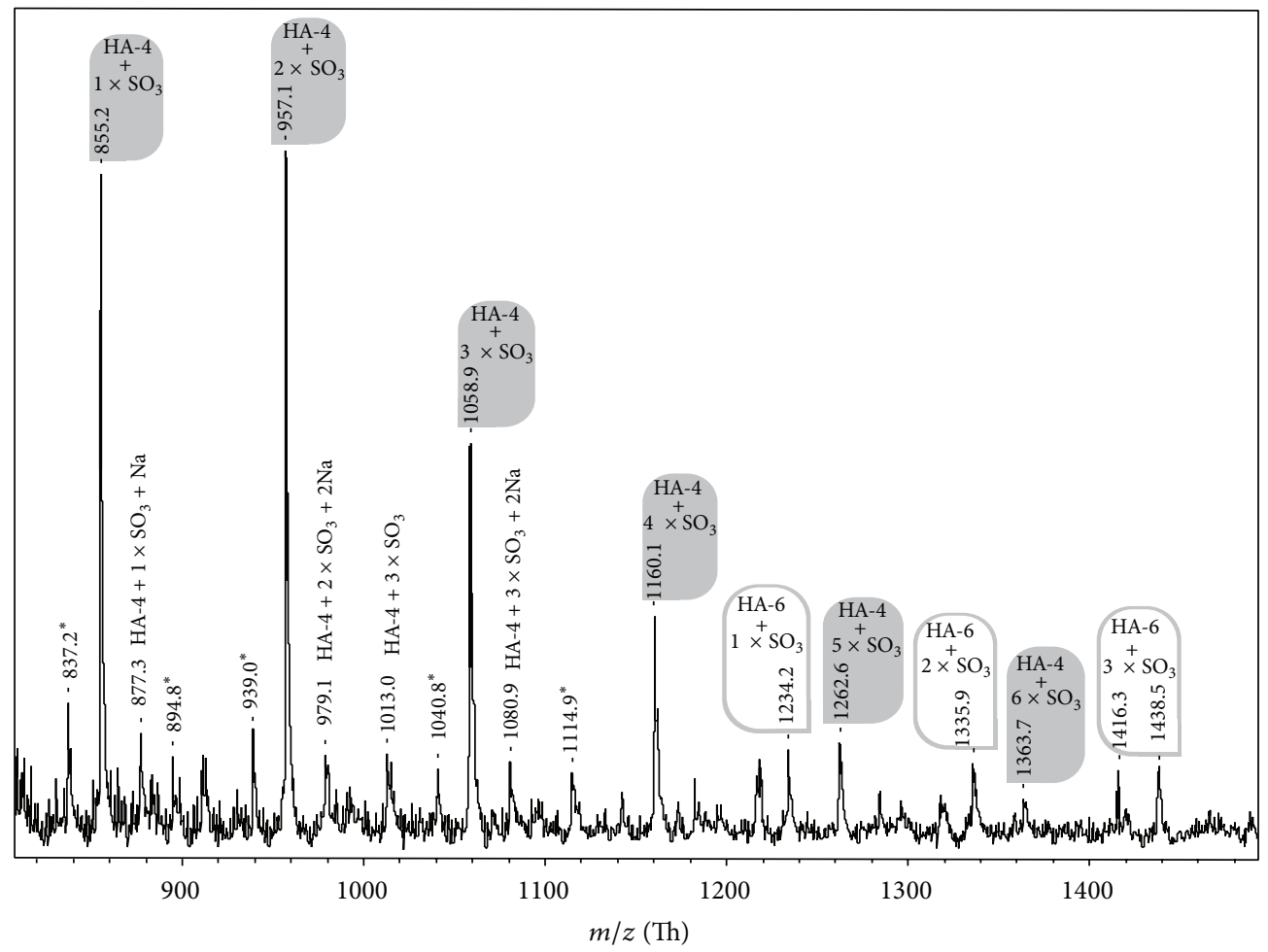

FIgURE 7: Negative ion MALDI-TOF mass spectrum of the sulfated HA with $\mathrm{ds}_{\mathrm{s}}=3.0$ subsequent to digestion with BTH at optimized conditions but without further purification of the reaction mixture. All spectra were recorded in the linear mode of the MS device and in the presence of 9 -aminoacridine as matrix using a sample to matrix ratio of 2 to $1(\mathrm{v} / \mathrm{v})$. Sulfated tetrasaccharides are marked by grey and hexasaccharides by white bars, respectively. Obvious impurities are marked by asterisks.

to our best knowledge it was so far not possible at all to convert highly sulfated HA samples into the corresponding oligosaccharides. Even if further improvements are obviously necessary, this study has provided sufficient evidence that strongly sulfated hyaluronans are basically digestible by bovine testicular hyaluronidase.

\section{Conclusions}

We have shown that chemically sulfated hyaluronan derivatives can be digested by bovine testicular hyaluronidase if the conditions of the enzymatic digestion are carefully adjusted, although the oligosaccharide yield is still strongly dependent on the number of sulfate residues per polymer repeating unit.

The molecular weights of the generated oligosaccharides were additionally verified by using MALDI-TOF mass spectrometry. All of the described experiments were exclusively performed on an analytical scale. However, our next step will be the extension of this study to obtain the oligosaccharides in at least mg amounts. If successful, this would be an important progress because the availability of GAG oligosaccharides with defined sulfation patterns is so far extremely limited.

\section{Conflict of Interests}

The authors declare that there is no conflict of interests regarding the publication of this paper.

\section{Acknowledgments}

The present research was supported by the German Research Council (Transregio 67, Projects A2 and A8). The authors are indebted to Mrs. Rosmarie Süß for many useful hints regarding the mass spectrometric characterization of sulfated glycosaminoglycans.

\section{References}

[1] J. Schiller and D. Huster, "New methods to study the composition and structure of the extracellular matrix in natural and bioengineered tissues," Biomatter, vol. 2, no. 3, pp. 115-131, 2012.

[2] B. Fuchs and J. Schiller, "Glycosaminoglycan degradation by selected reactive oxygen species," Antioxidants and Redox Signaling. In press.

[3] K. E. Benders, P. R. van Weeren, S. F. Badylak et al., "Extracellular matrix scaffolds for cartilage and bone regeneration," Trends in Biotechnology, vol. 31, no. 3, pp. 169-176, 2013.

[4] C. I. Gama and L. C. Hsieh-Wilson, "Chemical approaches to deciphering the glycosaminoglycan code," Current Opinion in Chemical Biology, vol. 9, no. 6, pp. 609-619, 2005.

[5] M. Schnabelrauch, D. Scharnweber, and J. Schiller, "Sulfated glycosaminoglycans as promising artificial extracellular matrix components to improve the regeneration of tissues," Current Medicinal Chemistry, vol. 20, no. 20, pp. 2501-2523, 2013.

[6] H. Liu, Z. Zhang, and R. J. Linhardt, "Lessons learned from the contamination of heparin," Natural Product Reports, vol. 26, no. 3, pp. 313-321, 2009. 
[7] J. Schiller, J. Becher, S. Möller, K. Nimptsch, T. Riemer, and M. Schnabelrauch, "Synthesis and characterization of chemically modified hyaluronan and chondroitin sulfate," Mini-Reviews in Organic Chemistry, vol. 7, no. 4, pp. 290-299, 2010.

[8] C. Hao, X. Ma, S. Fang et al., "Positive and negative-ion matrix-assisted laser desorption/ionization mass spectrometry of saccharides," Rapid Communications in Mass Spectrometry, vol. 12, no. 7, pp. 345-348, 1998.

[9] A. Nimptsch, S. Schibur, M. Schnabelrauch, B. Fuchs, D. Huster, and J. Schiller, "Characterization of the quantitative relationship between signal-to-noise $(\mathrm{S} / \mathrm{N})$ ratio and sample amount on-target by MALDI-TOF MS: determination of chondroitin sulfate subsequent to enzymatic digestion," Analytica Chimica Acta, vol. 635, no. 2, pp. 175-182, 2009.

[10] J. Schiller, J. Arnhold, S. Benard, S. Reichl, and K. Arnold, "Cartilage degradation by hyaluronate lyase and chondroitin $\mathrm{ABC}$ lyase: a MALDI-TOF mass spectrometric study," Carbohydrate Research, vol. 318, no. 1-4, pp. 116-122, 1999.

[11] I. Kakizaki, N. Ibori, K. Kojima, M. Yamaguchi, and M. Endo, "Mechanism for the hydrolysis of hyaluronan oligosaccharides by bovine testicular hyaluronidase," FEBS Journal, vol. 277, no. 7, pp. 1776-1786, 2010.

[12] A. Pichert, S. A. Samsonov, S. Theisgen et al., "Characterization of the interaction of interleukin-8 with hyaluronan, chondroitin sulfate, dermatan sulfate and their sulfated derivatives by spectroscopy and molecular modeling," Glycobiology, vol. 22, no. 1, pp. 134-145, 2012.

[13] C. Termeer, J. P. Sleeman, and J. C. Simon, "Hyaluronan-magic glue for the regulation of the immune response?" Trends in Immunology, vol. 24, no. 3, pp. 112-114, 2003.

[14] T. Toida, Y. Ogita, A. Suzuki, H. Toyoda, and T. Imanari, "Inhibition of hyaluronidase by fully $\mathrm{O}$-sulfonated glycosaminoglycans," Archives of Biochemistry and Biophysics, vol. 370, no. 2, pp. 176-182, 1999.

[15] D. J. Langeslay, C. J. Jones, S. Beni, and C. K. Larive, "Glycosaminoglycans: oligosaccharide analysis by liquid chromatography, capillary electrophoresis, and specific labeling," Methods in Molecular Biology, vol. 836, pp. 131-144, 2012.

[16] V. Hintze, S. Moeller, M. Schnabelrauch et al., "Modifications of hyaluronan influence the interaction with human bone morphogenetic protein-4 (hBMP-4)," Biomacromolecules, vol. 10, no. 12, pp. 3290-3297, 2009.

[17] J. L. Reissig, J. L. Storminge, and L. F. Leloir, "A modified colorimetric method for the estimation of N-acetylamino sugars," The Journal of Biological Chemistry, vol. 217, no. 2, pp. 959-966, 1955.

[18] I. Muckenschnabel, G. Bernhardt, T. Spruss, B. Dietl, and A. Buschauer, "Quantitation of hyaluronidases by the MorganElson reaction: comparison of the enzyme activities in the plasma of tumor patients and healthy volunteers," Cancer Letters, vol. 131, no. 1, pp. 13-20, 1998.

[19] T. Asteriou, B. Deschrevel, B. Delpech et al., "An improved assay for the $\mathrm{N}$-acetyl-D-glucosamine reducing ends of polysaccharides in the presence of proteins," Analytical Biochemistry, vol. 293, no. 1, pp. 53-59, 2001.

[20] S. Nagaraju, K. S. Girish, Y. Pan, K. A. Easely, and K. Kemparaju, "Estimation of serum hyaluronidase activity overcoming the turbidity interference," Clinical Laboratory Science, vol. 24, no. 3, pp. 172-177, 2011.
[21] P. B. O'Connor, K. Dreisewerd, K. Strupat, and F. Hillenkamp, "MALDI mass spectrometry instrumentation," in MALDI MSA Practical Guide to Instrumentation, Methods, and Applications, F. Hillenkamp and J. Peter-Katalinic, Eds., pp. 41-104, Wiley-VCH, Weinheim, Germany, 2nd edition, 2013.

[22] K. Busse, M. Averbeck, U. Anderegg, K. Arnold, J. C. Simon, and J. Schiller, "The signal-to-noise ratio as a measure of HA oligomer concentration: a MALDI-TOF MS study," Carbohydrate Research, vol. 341, no. 8, pp. 1065-1070, 2006.

[23] G. Sun, K. Yang, Z. Zhao, S. Guan, X. Han, and R. W. Gross, "Matrix-assisted laser desorption/ionization time-offlight mass spectrometric analysis of cellular glycerophospholipids enabled by multiplexed solvent dependent analyte-matrix interactions," Analytical Chemistry, vol. 80, no. 19, pp. 75767585, 2008.

[24] K. Nimptsch, R. Süß, M. Schnabelrauch, A. Nimptsch, and J. Schiller, "Positive ion MALDI-TOF mass spectra are more suitable than negative ion spectra to characterize sulphated glycosaminoglycan oligosaccharides," International Journal of Mass Spectrometry, vol. 310, pp. 72-76, 2012.

[25] N. Volpi, J. Schiller, R. Stern, and L. Šoltés, "Role, metabolism, chemical modifications and applications of hyaluronan," Current Medicinal Chemistry, vol. 16, no. 14, pp. 1718-1745, 2009.

[26] R. Stern, G. Kogan, M. J. Jedrzejas, and L. Šoltés, “The many ways to cleave hyaluronan," Biotechnology Advances, vol. 25, no. 6, pp. 537-557, 2007.

[27] “Enzyme Nomenclature," Academic Press, San Diego, Calif, USA, 1992, http://www.chem.qmul.ac.uk/iubmb/enzyme.

[28] E. Sisu, C. Flangea, A. Serb, and A. D. Zamfir, "Modern developments in mass spectrometry of chondroitin and dermatan sulfate glycosaminoglycans," Amino Acids, vol. 41, no. 2, pp. 235-256, 2011.

[29] J. Zaia, "Glycosaminoglycan glycomics using mass spectrometry," Molecular and Cellular Proteomics, vol. 12, no. 4, pp. 885892, 2013.

[30] T. N. Laremore and R. J. Linhardt, "Improved matrix-assisted laser desorption/ionization mass spectrometric detection of glycosaminoglycan disaccharides as cesium salts," Rapid Communications in Mass Spectrometry, vol. 21, no. 7, pp. 1315-1320, 2007.

[31] J. Kovensky, "Sulfated oligosaccharides: new targets for drug development?" Current Medicinal Chemistry, vol. 16, no. 18, pp. 2338-2344, 2009. 

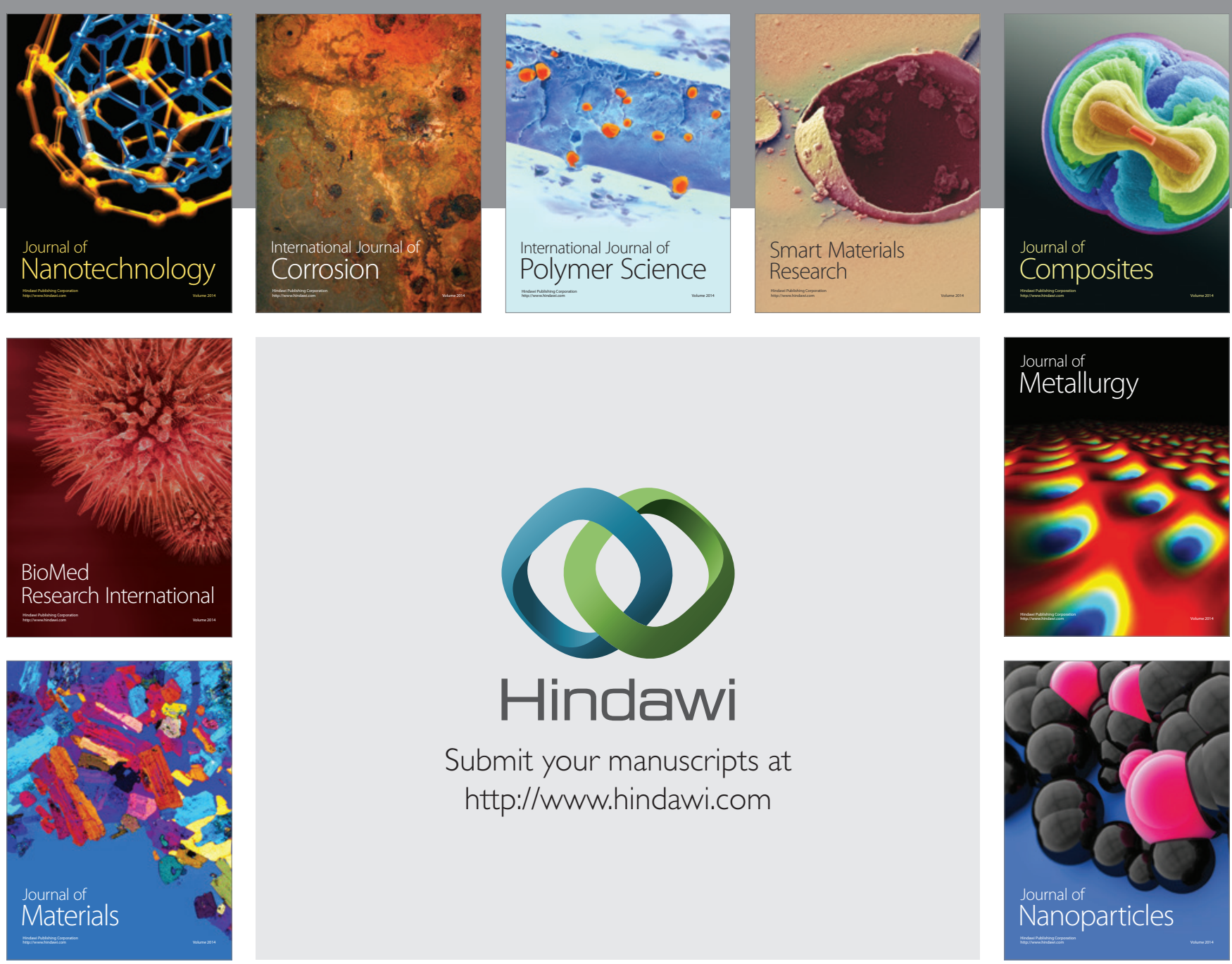

Submit your manuscripts at http://www.hindawi.com
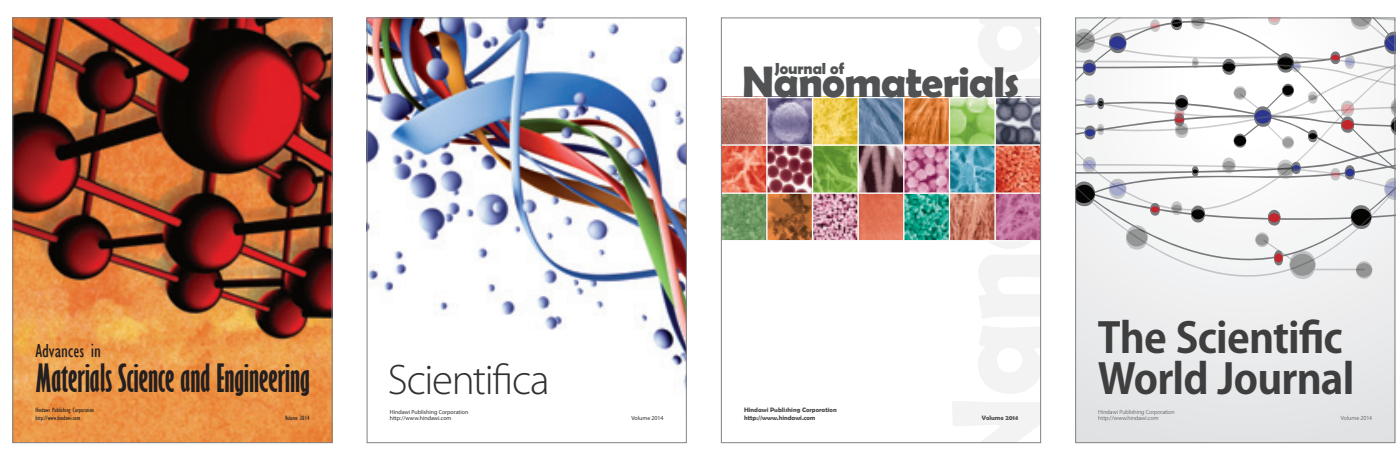

\section{The Scientific World Journal}
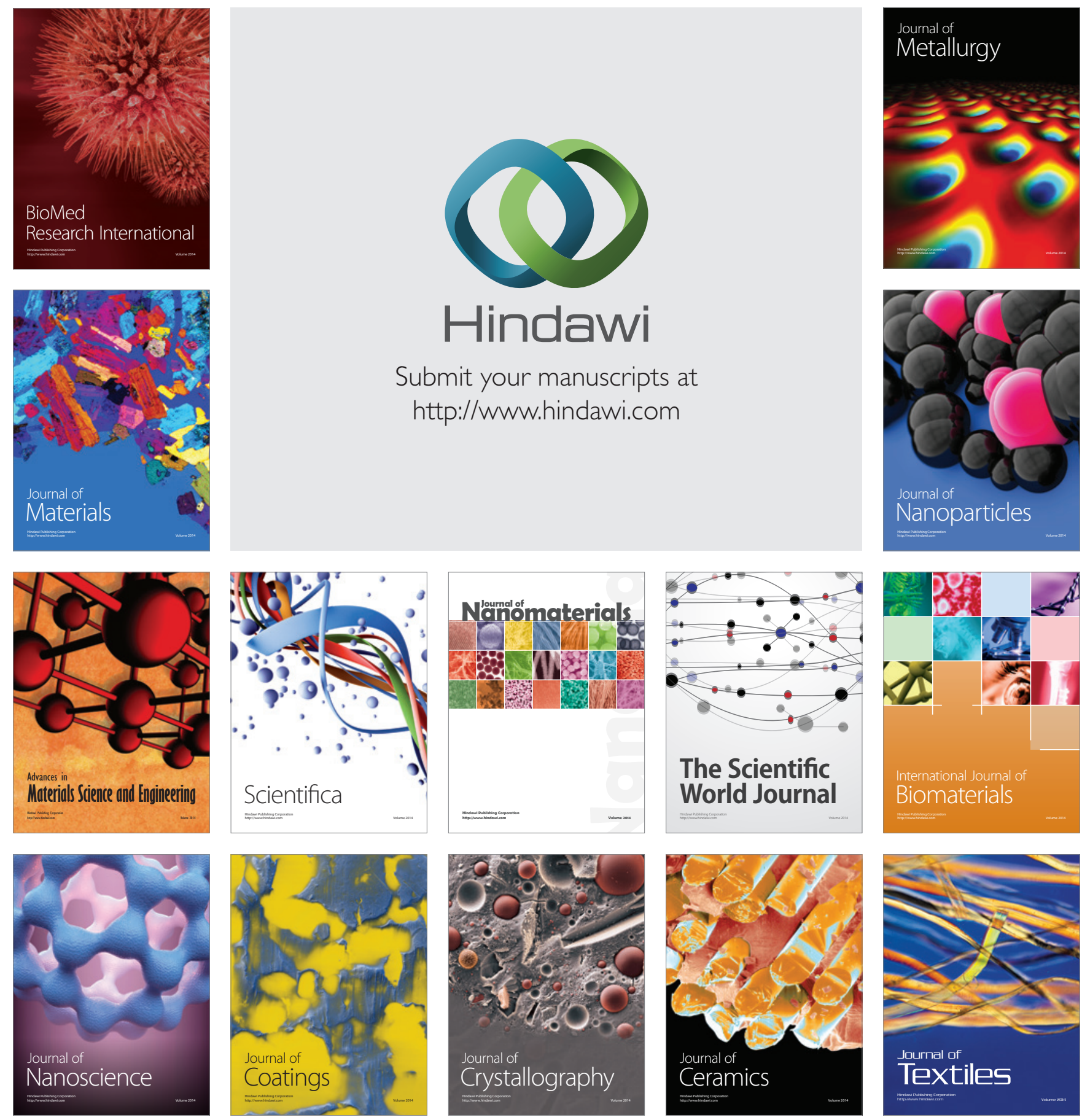